\title{
Addition of Diffusion-Weighted Imaging to Dynamic Contrast Enhanced MRI: can it help differentiate benign from malignant jaw lesions?
}

Xue Zhao ( zhaoxuecomeon@sina.com )

Research article

Keywords: Magnetic Resonance Imaging, Diffusion Weighted Imaging, Apparent Diffusion Coefficients

Posted Date: January 17th, 2020

DOI: https://doi.org/10.21203/rs.2.21207/v1

License: (a) (i) This work is licensed under a Creative Commons Attribution 4.0 International License.

Read Full License 


\section{Abstract}

Objective: To explore the diagnosis value of adding diffusion-weighted imaging (DWI) to dynamic contrast enhanced MRI (DCE-MRI) in distinguishing benign from malignant jaw lesions.

Materials and Methods: This retrospective study was involved 53 patients ( 23 benign, 30 malignant) with jaw lesions confirmed by pathology were analyzed. DWI and DCE-MRI were performed in all patients. The Apparent Diffusion Coefficient (ADC) value as well as the DCE-MRI parameters [time to peak $\left(T_{\text {peak }}\right)$, wash in rate (WIR), wash out rate (WOR), relative enhancement, and maximum enhancement] were measured for each patient. The optimal cut-off value of ADC values and DCE-MRI parameters were determined by using a receiver operating characteristics (ROCs), and the area under ROC curve (AUC) was evaluated. $P<$ 0.05 was considered to indicate a significant difference.

Results: Among the five parameters of DCE-MRI, the WOR displayed the most significantly difference with a threshold value of $4.90 \mathrm{l} / \mathrm{s}$ between benign and malignant group $(P<0.05)$. When only WOR was used as the basis for diagnosis, the sensitivity, specificity, and AUC were $77.20 \%, 78.00 \%$, and 0.800 , respectively (Fig. 3 , 4). However, sensitivity $(77.20 \%$ vs $83.30 \%)$, specificity $(78.00 \%$ vs $87.20 \%)$ and accuracy $(0.80$ vs 0.85 ) significantly improved with the addition of ADC values in the evaluation of jaw lesions, which manifested better than that by using WOR alone.

Conclusion: The high WOR of DCE-MRI may have a malignant tendency, but in the evaluation of jaw disease, the addition of $A D C$ value can improve the diagnostic value.

\section{Background}

Imaging of lesions within the maxilla and mandible is often fraught with difficulty owing to the similarity in the imaging appearance of a diverse array of pathological processes ${ }^{(1,2)}$. Array of lesions with the jaw reveal similar localization features, most of which arising in the posterior body and lower ramus of the mandible $e^{(3,4)}$. Even though conventional imaging, such as computed tomography (CT) and magnetic resonance imaging (MRI) can represent the pertinent reference such as the location, relationship to the tooth, margins of the lesion, size of the lesion and solid or cystic performance of the lesion, which of the imaging findings can narrow the differential diagnosis, many lesions still often exist considerable conventional imaging overlap ${ }^{(2,5-7)}$. Assessing the biological behavior among the mandible and maxilla lesions is the first step for clinical diagnosis and management. ${ }^{(8)}$ Previous studies involving the roles of functional MRI, such as diffusion-weighted imaging (DWI) and dynamic contrast- enhanced (DCE) MRI already have a wild application $(7,9,10)$, such as the brain, prostate, and breast, and head and neck ${ }^{(11-13)}$. The usefulness of DWI has been reported ${ }^{(14,15)}$, and the type of time-intensity curve (TIC) generated by DCE-MRI is of a great value as well ${ }^{(16)}$. However, these are not as accurate as quantitative or semiquantitative parameters. In this study, we try to introduce array of semi-quantitative parameters measured by DCE-MRI \such as wash in rate(WIR), time to peak $\left(T_{\text {peak }}\right)$, wash out rate (WOR). To our knowledge, the exploration of the combination of DWI and DCE-MRI in the jaw lesions has not been emphasized 
previously in the literature. Therefore, our study aims to determine whether the addition of DWI to DCEMRI can help distinguish between benign and malignant jaw lesions.

\section{Methods}

Our retrospective study was approved by the institutional review committee of the Shanghai Ninth People's Hospital and the informed consent requirement was waived. A total of 53 patients (29 male, 24 female; mean age, 41.5 years) were enrolled between January 2017 and May 2019. All patients were examined with gadolinium-enhanced dynamic MR imaging and DWI at our hospital. Histopathologic diagnoses were made on the basis of findings in specimens obtained at surgical resection. The exclusion criteria were as follows: (1) with interfering the diagnosis; (2) with a previously diagnosed head and neck cancer; or (3) with treatment of a head and neck lesion (surgical management or radiotherapy) before the MRI scan.

MRI examinations were performed within 2 weeks before surgery in all patients. All MRI examinations were performed on a 3.0-T imaging system (Philips Achieva; Philips Medical Systems, Best, the Netherlands) with a head and neck array coil. Pre- and post contrast transverse T1-weighted spin-echo (repetition time msec/echo time msec, 659/10; $256 \times 256$ matrix; 5-mm section thickness; 1-mm intersection gap; 24-24-cm field of view; one signal acquired) and transverse T2-weighted fast spin-echo (repetition time msec/echo time msec, 3200/100; $256 \times 256$ matrix; 5-mm section thickness; 2.5-mm intersection gap; $20-25-\mathrm{cm}$ field of view; one signal acquired) MR images were obtained. DWI was performed using a single-shot spin echo echoplanar imaging sequence (TR/TE, 2775/70 ms; matrix, 128 $\times 128 \mathrm{~mm}$; FOV, $240 \times 240 \mathrm{~mm}$; thickness/gap, 5.0/0.5 mm; b, 0 and $800 \mathrm{~s} \mathrm{~mm}^{-2} ; \mathrm{x}-\mathrm{y}$-, and z-gradient directions). ADC maps were generated. DCE-MRI was obtained after intravenous injection of gadopentetate dimeglumine (Magnevist ${ }^{\circledR}$; Schering, Berlin, Germany) at a dosage of $0.1 \mathrm{mmol} \mathrm{kg}^{-1}$ of body weight (TR/TE, 4.8/2.2 ms; FOV, $240 \times 240$ mm; matrix, $256 \times 192$ mm; thickness/gap, 5.0/0 mm).

Two radiologists selected representative regions of interest (ROIs) by consensus (X.Z. and J.B.W., with 5 and 10 years of experience in maxillofacial imaging and contrast-enhanced MRI, respectively). For ADC measurements, freehand ROI was drawn, taking care to exclude obvious necrotic or non-perfused areas by visual correlation with pre- and post-contrast $\mathrm{T} 1$ weighted images and to avoid the most peripheral portions because of partial volume effects of adjacent tissue. Three ROIs were manually placed within each lesion, and the averaged ADC value was obtained. For post-processing of DCE-MRI, we placed ROIs (approximately 25-50 $\mathrm{mm}^{2}$ ) over the lesion and time-intensity curve (TIC) for each ROI was generated. The TIC (axis co-ordinate, time; vertical, signal intensity) with the most prominent enhancement was selected as the representation for each lesion. The following DCE-MRI parameters were automatically calculated for each patient: $T_{\text {peak }}$ (time to enhancement peak minus the time at which the maximum slope tangent intersects the $x$-axis), relative enhancement (the difference between post - pre enhancement on the $y$-axis), maximum enhancement (time to peak enhancement extend the value at which the maximum slope tangent intersects the $y$-axis), wash in rate (maximum slope, tangent at the 
ascending part of the time-intensity curve), wash out rate (minimum slope, tangent at the descending part of the time-intensity curve).

\section{Statistical analysis}

Statistical analysis was carried out using SPSS 23.0 (2012, Chicago, USA). Descriptive statistics, such as the age of male and female patients, and disease duration in patients, were compared with MannWhitney $\mathrm{U}$ test for independent samples. 95\% confidence intervals (Cls) were calculated using logistic regression models to determine the association of DCE-MRI parameters between benign and malignant jaw, as well as the ADC value. To find the feasible threshold of ADC value and DCE-MRI parameters to distinguish malignant from benign lesions, we conducted receiver operator characteristics (ROCs) analysis. We plotted the sensitivity versus 1-specificity for each cut-off value to generate the ROC curve, and the areas under the ROC curve (AUC) were assessed. We then conducted and compared the AUC of the DCE-MRI parameters, then add the ADC value to the most significantly parameter of DEC-MRI to evaluate the diagnostic ability. $p<0.05$ was considered as statistically significant.

\section{Results}

In 23 of the 53 patients, malignant jaw lesions were diagnosed as gingiva squamous cell carcinoma invading jaw (fig.1) ( $n=12)$, primary intraosseous squamous cell carcinoma $(n=8)$, and ameloblastic carcinoma $(n=3)$. The remaining 30 patients with benign jaw lesions were diagnosed as ameloblastomasolid type (fig.2) ( $n=17)$, adenomatoid odontogenic tumor ( $n=7)$, odontogenic myxoma $(n=6)$. In no statistical difference was found in sex and age of patients with benign versus malignant lesions of mandible and maxilla $(P>0.05)$.

Among the five parameters of DCE-MRI, benign group $(n=23)$ and malignant group $(n=30)$ differed in $T_{\text {peak }}$, WIR, and WOR $(P<0.05)$, and the WOR displayed the most significantly difference (Table 1, fig.3-6). Malignant group showed higher WOR, WIR, but lower $T_{\text {peak }}$ and ADC value compared with the benign group $(p<0.05)(f i g .6-9)$. The optimal cut-off value of WOR value was $4.9 \mathrm{l} / \mathrm{s}$ (Table 2), which suggested a malignant diagnosis with $\mathrm{WOR}>4.9 \mathrm{l} / \mathrm{s}$ and a benign diagnosis with $\mathrm{WOR} \leq 4.9 \mathrm{l} / \mathrm{s}$. Maximum or relative enhancement is not helpful to distinguish benign and malignant lesions $(P>0.05)$. In addition to DCE-MRI parameters, the $A D C$ value displayed a threshold value of $1.05 \times 10^{-3} \mathrm{~mm}^{2} / \mathrm{s}$ (Table 2), which suggested a malignant diagnosis with $A D C$ value $\leq 1.05 \times 10^{-3} \mathrm{~mm}^{2} / \mathrm{s}$ (Fig 1 ) and a benign diagnosis with $A D C$ value $>1.05 \times 10^{-3} \mathrm{~mm}^{2} / \mathrm{s}$ (Fig 2).

When only WOR was used as the basis for diagnosis, the sensitivity, specificity, and AUC were $77.2 \%$, $78.0 \%$, and 0.80 , respectively(fig.7-8). However, sensitivity (77.2\% vs $83.3 \%)$, specificity $(78.0 .0 \%$ vs $87.2 \%$ ) and accuracy ( 0.80 vs 0.85 ) significantly improved with the addition of ADC values in the evaluation of jaw lesions(fig.9), which manifested better than that by using WOR alone (Table2).

\section{Discussion}


Our findings show that adding DWI to DCE-MRI can improve diagnostic value in mandible and maxilla tumors. With the addition of ADC, it may be possible to avoid missing cancers and performing unnecessary surgery or biopsy for benign lesions in the jaw. DWI quantifies the diffusional mobility of water protons with apparent diffusion coefficient (ADC). DCE-MRI provides information related to the physiology of the microcirculation and vascularity. As with most DWI applications, tumor cell density is higher, and more cell membranes theoretically have a greater impediment to diffusion, consequently, a lower ADC. On the contrary, $A D C$ is higher . Given the heterogeneous group of benign and malignant lesions, there will clearly be exceptions and overlap in ADC value as well as the WOR results. A single parameter threshold cannot be used in all circumstances, and the establishment of threshold for specific diagnosis is required. Among a wild range of head and neck lesions, previous studies have established an optimal ADC threshold of $1.3 \times 10^{-3} \mathrm{~mm}^{2} / \mathrm{s}$ for diagnosis between benign and malignant lesions ${ }^{(17)}$, and the $A D C$ value of malignant tumors, benign solid masses, and cystic lesions of $0.93 \times 10^{-3} \mathrm{~mm}^{2} / \mathrm{s} \pm 0.18$, $1.57 \times 10^{-3} \mathrm{~mm}^{2} / \mathrm{s} \pm 0.26$, and $2.01 \times 10^{-3} \mathrm{~mm}^{2} / \mathrm{s} \pm 0.21$, respectively ${ }^{(18)}$. For specific mandible and maxilla lesions, our results found the $A D C$ value of benign and malignant group of $1.55 \times 10^{-3} \mathrm{~mm}^{2} / \mathrm{s} \pm$ $0.83,0.95 \times 10^{-3} \mathrm{~mm}^{2} / \mathrm{s} \pm 0.37$, respectively, which was more specific than the previous reports.

$T_{\text {peak }}$ is believed to represent microvascular counts, and in head and neck tumors, the threshold between benign and malignant tumors has been described as about 120 seconds $^{(19)}$. However, there is no clear data on the identification of specific jaw lesions. The data from this group showed a threshold of 75.78 seconds for the "benign and malignant tumors" group, which is more suitable for maxillary and mandibular lesions than previously reported. WOR represents a difference in medium concentration between the intravascular and extravascular phases; tumors with high cellularity exhibit higher wash out rate values ${ }^{(20)}$. Previous authors reported that enhanced areas of benign lesion-fibroblastoma showed a gradual enhancement on DCE-MRI, which corresponded to solid components and/or intramural nodules, and the solid area reached 600 After s gradually disappears or maintains an enhanced level. The maximum enhancement level, our results showed that the mean WOR of benign lesions was $4.09 \mathrm{I} / \mathrm{s} \pm$ 6.10 , while in the malignant group, the mean WOR was $21.4 \mathrm{I} / \mathrm{s} \pm 31.37$, significantly higher than the benign group. Due to the high cell density in malignant tumors, the WOR threshold for benign and malignant groups was $4.9 \mathrm{I}$ / s, corresponding to previous studies. Other parameters of DCE-MRI in our study, including maximal enhancement and relative enhancement, did not reveal significant differences in the identification of benign and malignant lesions of the jaw. By adding the ADC value, the sensitivity, specificity, and accuracy are significantly improved, which is better than using WOR alone. In addition, because DWI combined with DCE-MRI can improve the diagnostic accuracy, whether traditional MRI combined with DWI and DCE-MRI can improve the diagnostic accuracy is still to be further exploration.

Our study had the following limitations: First, due to the low incidence rate and the strict exclusion criteria of the study, we included a relatively small number of jaw lesions, thereby further prospective and multicenter studies need to validate our findings. Second, the reproducibility of ADC values between MR pulse sequences remains controversial. As such, the cutoff level for the ADC value derived from our results might not be transferable to other institutions. In addition, given the heterogeneous origin of 
benign and malignant lesions with jaw, data from larger studies are required to define in specific pathological entities.

In conclusion, the high WOR of DCE-MRI may have a malignant tendency, but in the evaluation of jaw disease, the addition of ADC value can improve the diagnostic value. DWI combined with DCE-MRI parameters can better distinguish benign and malignant lesions in the mandible than single parameters, which is of great significance for preoperative diagnosis of jaw lesions.

\section{Declarations}

Acknowledgements

Not applicable

Funding

This work was supported by funds from the National Scientific Foundation of China (No.91859202, 8177901)

Authors' contributions

Xue Zhao contributed to the study design, the collection and assembly of data and drafted the manuscript, Jingbo Wang did the feature extraction statistical work and prepared figures. All the authors read and approved the final manuscript.

Ethics approval and consent to participate

Our retrospective study was approved by the institutional review committee of the Shanghai Ninth People's Hospital and the informed consent requirement was waived.

Consent for publication

Not applicable.

Competing interests

The author declare that there have no competing interests.

Author details

Department of Radiology, Shanghai Ninth People's Hospital, Shanghai JiaoTong University School of Medicine,

Shanghai, 200011, China;

Email: zhaoxuecomeon@sina.com 


\section{Abbreviations}

DWI= adding diffusion-weighted imaging, $\mathrm{MRI}=$ magnetic resonance imaging, DCE-MRI= dynamic contrast enhanced MRI, ADC= apparent diffusion coefficient, $\mathrm{WOR}=$ wash out rate, $T_{\text {peak }}=$ time to peak, $\mathrm{WIR}=$ wash in rate $\mathrm{TIC}=$ time-intensity curve, $\mathrm{ROC}=$ receiver operating characteristic, $\mathrm{AUC}=$ area under receiver operating characteristic curve, $\mathrm{ROI}=$ region of interest

\section{References}

1. Slootweg PJ. Lesions of the jaws. Histopathology 2009;54:401-418

2. Mosier KM. Lesions of the Jaw. Semin Ultrasound CT MR 2015;36:444-450

3. Avril L, Lombardi T, Ailianou A, Burkhardt K, Varoquaux A, Scolozzi P, et al. Radiolucent lesions of the mandible: a pattern-based approach to diagnosis. Insights Imaging 2014;5:85-101

4. Harmon $\mathrm{M}$, Arrigan $\mathrm{M}$, Toner $\mathrm{M}$, O'Keeffe SA. A radiological approach to benign and malignant lesions of the mandible. Clin Radiol 2015;70:335-350

5. Weber AL, Bui C, Kaneda T. Malignant tumors of the mandible and maxilla. Neuroimaging Clin N Am 2003;13:509-524

6. Mosier KM. Magnetic resonance imaging of the maxilla and mandible: signal characteristics and features in the differential diagnosis of common lesions. Top Magn Reson Imaging 2015;24:23-37

7. Van Cann EM, Rijpkema M, Heerschap A, van der Bilt A, Koole R, Stoelinga PJ. Quantitative dynamic contrast-enhanced MRI for the assessment of mandibular invasion by squamous cell carcinoma. Oral Oncol 2008;44:1147-1154

8. Dutta M, Kundu S, Bera H, Barik S, Ghosh B. Ameloblastic carcinoma of mandible: facts and dilemmas. Tumori 2014;100:e189-196

9. Hara M, Matsuzaki H, Katase N, Yanagi Y, Unetsubo T, Asaumi J, et al. Central odontogenic fibroma of the jawbone: 2 case reports describing its imaging features and an analysis of its DCE-MRI findings. Oral Surg Oral Med Oral Pathol Oral Radiol 2012;113:e51-58

10. Hara M, Matsuzaki H, Katase N, Yanagi Y, Unetsubo T, Asaumi J, et al. Ossifying fibroma of the maxilla: a case report including its imaging features and dynamic magnetic resonance imaging findings. Oral Surg Oral Med Oral Pathol Oral Radiol 2012;114:e139-146

11. Thoeny HC, De Keyzer F, King AD. Diffusion-weighted MR imaging in the head and neck. Radiology 2012;263:19-32

12. Rumley CN, Lee MT, Holloway L, Rai R, Min M, Forstner D, et al. Multiparametric magnetic resonance imaging in mucosal primary head and neck cancer: a prospective imaging biomarker study. $B M C$ Cancer 2017;17:475

13. Yanagi Y, Asaumi J, Unetsubo T, Ashida M, Takenobu T, Hisatomi M, et al. Usefulness of MRI and dynamic contrast-enhanced MRI for differential diagnosis of simple bone cysts from true cysts in the jaw. Oral Surg Oral Med Oral Pathol Oral Radiol Endod 2010;110:364-369 
14. Wang J, Takashima S, Takayama F, Kawakami S, Saito A, Matsushita T, et al. Head and neck lesions: characterization with diffusion-weighted echo-planar MR imaging. Radiology 2001;220:621-630

15. Eida S, Sumi M, Sakihama N, Takahashi H, Nakamura T. Apparent diffusion coefficient mapping of salivary gland tumors: prediction of the benignancy and malignancy. AJNR Am J Neuroradiol 2007;28:116-121

16. Yabuuchi H, Matsuo Y, Kamitani T, Setoguchi T, Okafuji T, Soeda H, et al. Parotid gland tumors: can addition of diffusion-weighted MR imaging to dynamic contrast-enhanced MR imaging improve diagnostic accuracy in characterization? Radiology 2008;249:909-916

17. Srinivasan A, Dvorak R, Perni K, Rohrer S, Mukherji SK. Differentiation of benign and malignant pathology in the head and neck using 3T apparent diffusion coefficient values: early experience. AJNR Am J Neuroradiol 2008;29:40-44

18. Abdel Razek AA, Gaballa G, Elhawarey G, Megahed AS, Hafez M, Nada N. Characterization of pediatric head and neck masses with diffusion-weighted MR imaging. Eur Radio/ 2009;19:201-208

19. Chikui T, Obara M, Simonetti AW, Ohga M, Koga S, Kawano S, et al. The principal of dynamic contrast enhanced MRI, the method of pharmacokinetic analysis, and its application in the head and neck region. Int J Dent 2012;2012:480659

20. Kitamoto E, Chikui T, Kawano S, Ohga M, Kobayashi K, Matsuo Y, et al. The application of dynamic contrast-enhanced MRI and diffusion-weighted MRI in patients with maxillofacial tumors. Acad Radiol 2015;22:210-216

\section{Tables}

Table 1 Quantitative parameters in benign and malignant group of DWI and DCE-MRI.

\begin{tabular}{|c|c|c|c|c|c|}
\hline \multirow[b]{2}{*}{ parameter } & \multicolumn{2}{|c|}{ Benign } & \multicolumn{2}{|c|}{ Malignant } & \multirow[b]{2}{*}{$P$ Value } \\
\hline & mean $\pm \mathrm{SD}$ & $95 \% \mathrm{CI}$ & mean $\pm S D$ & $95 \% \mathrm{CI}$ & \\
\hline Relative enhancement & $106.31 \pm 54.89$ & $81.97 \square 130.65$ & $111.50 \pm 47.00$ & $92.10 \square 130.90$ & 0.728 \\
\hline Maximum enhancement & $847.27 \pm 413.76$ & $663.82 \llbracket 1030.72$ & $990.28 \pm 458.21$ & $801.13 \square 1179.42$ & 0.27 \\
\hline $\mathrm{T}_{\text {peak }}$ & $113.65 \pm 26.50$ & $0.30,39.64$ & $93.69 \pm 38.45$ & $77.82 \llbracket 109.56$ & 0.047 \\
\hline WIR & $46.17 \pm 27.45$ & $33.98 \square 58.32$ & $93.69 \pm 38.45$ & $77.82 \llbracket 109.55$ & 0.02 \\
\hline WOR & $4.09 \pm 6.10$ & $1.39 \square 6.79$ & $21.40 \pm 31.37$ & $8.45,34.35$ & 0.011 \\
\hline ADC value & $1.55 \pm 0.83$ & $1.18,1.92$ & $0.95 \pm 0.37$ & $0.80,1.10$ & 0.009 \\
\hline
\end{tabular}

Note: $\mathrm{CI}=$ confidence interval, $\mathrm{SD}=$ standard deviation.

Table2 Areas under Curve, Sensitivity, and Specificity of parameters to Differentiate benign from malignan 


\begin{tabular}{lllll}
\hline Parameter & AUC & Cut-off Value & Sensitivity $\%$ 口 & Specificity (\%) \\
\hline $\mathrm{T}_{\text {peak }}$ & $0.66(0.50,0.82)$ & $<75.78$ & $62.60(0.39,0.74)$ & $60.10(0.30,0.81))$ \\
WIR & $0.71(0.56,0.85)$ & $<42.27$ & $70.0(0.46,0.85)$ & $62.50(0.40,0.80)$ \\
WOR & $0.80(0.58,0.89)$ & $<4.29$ & $77.20(0.46,0.89)$ & $78.00(0.35,0.76)$ \\
ADC value & $0.81(0.62,0.93)$ & $<1.05$ & $76.80(0.51,0.84)$ & $85.60(0.56,0.97)$ \\
& & & & \\
WOR+ADC value & $0.85(0.69,0.94)$ & $/$ & $83.30(0.59,0.91)$ & $87.20(0.60,0.96)$ \\
& & & & \\
\hline
\end{tabular}

\section{Figures}



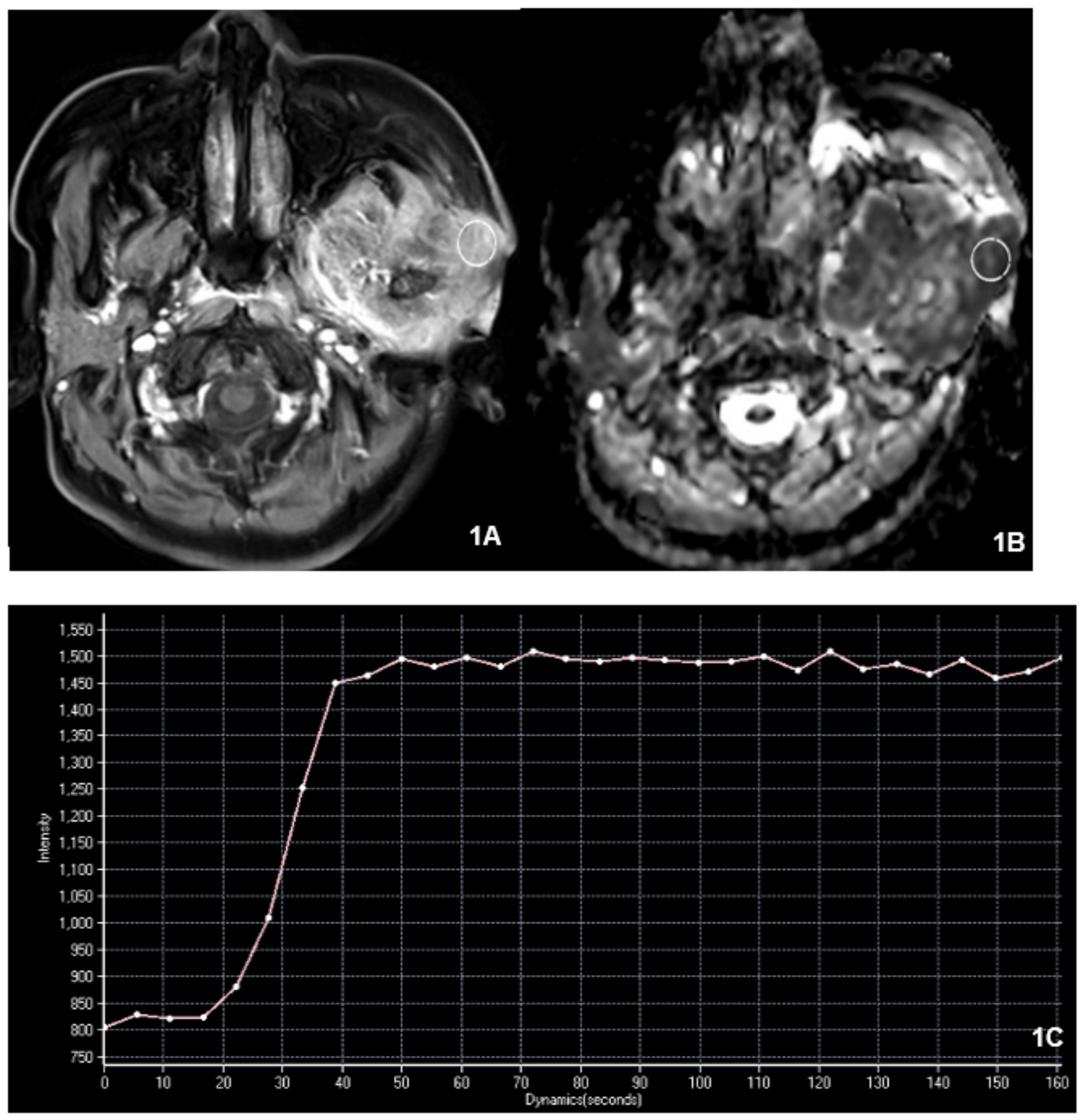

\section{Figure 1}

A 42-year-old female presented with gingiva squamous cell carcinoma invading jaw at left mandibular region. Fig. A. Axial DCE-MRI image displayed the gingiva squamous cell carcinoma invading jaw at left mandibular region with enhancement. Time intensity curve (TIC) was measured at dynamic-contrast MRI. The round cursors mark the regions of interest. Fig. $B$. The apparent diffusion coefficient (ADC) value is $0.6 \times 10-3 \mathrm{~mm} 2 / \mathrm{s}$ in ADC map. Fig. C. The time intensity curve (TIC) demonstrated the Tpeak was $60.95 \mathrm{~s}$, wash in rate was $69.80 \mathrm{l} / \mathrm{s}$, and washout rate was $6.14 \mathrm{l} / \mathrm{s}$. 

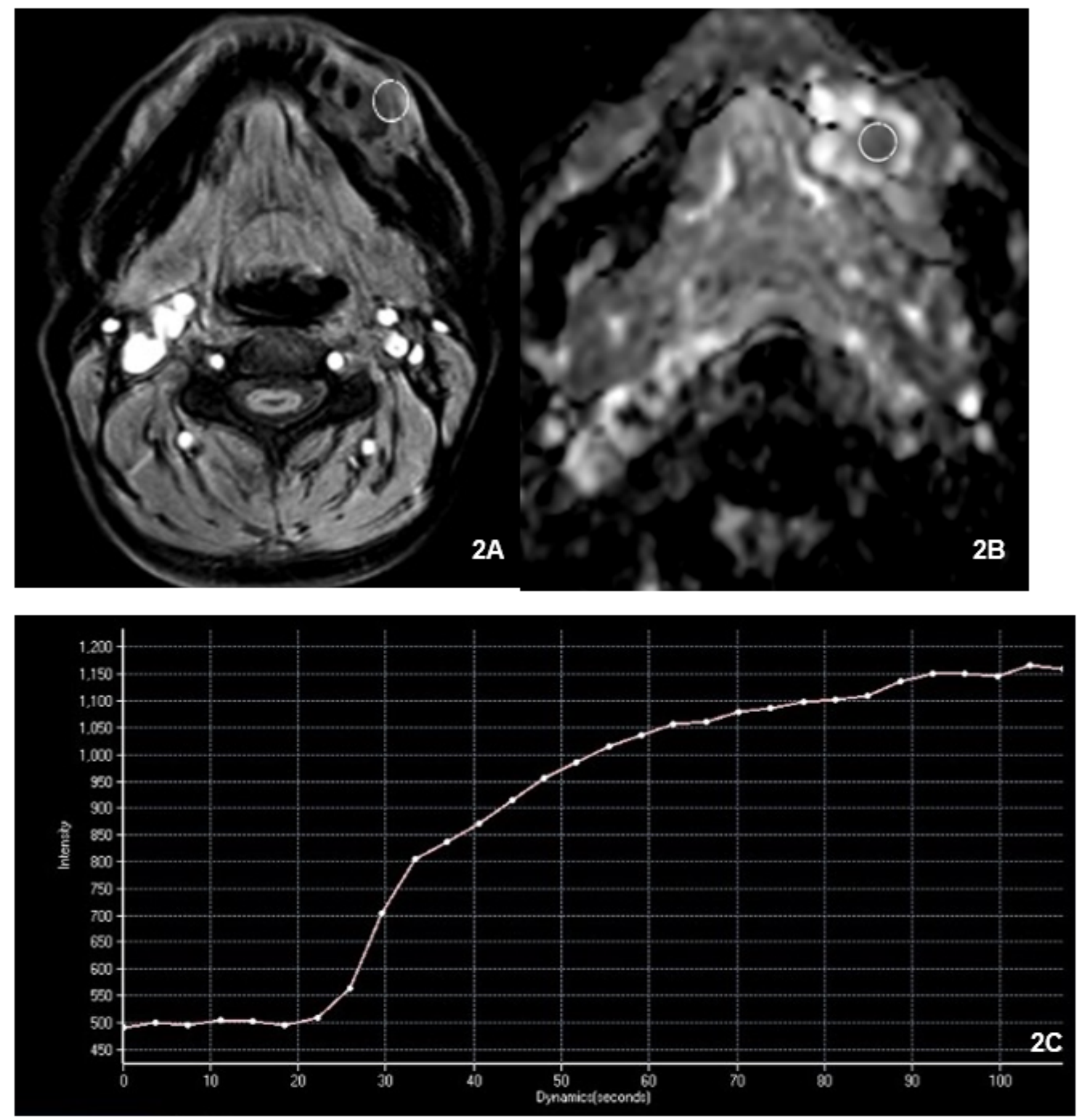

Figure 2

A 45-year-old female presented with ameloblastoma-solid type of the left mandible. Fig. A. Axial DCE-MRI image displayed the ameloblastoma at left mandibular region with enhancement. Time intensity curve (TIC) was measured at dynamic-contrast MRI. The round cursors mark the regions of interest. Fig. B.The apparent diffusion coefficient (ADC) value is $1.5 \times 10-3 \mathrm{~mm} 2 / \mathrm{s}$ in ADC map. Fig. C. TIC demonstrated the Tpeak was $103.45 \mathrm{~s}$, wash in rate was $19.84 \mathrm{l} / \mathrm{s}$, and washout rate was $0.95 \mathrm{l} / \mathrm{s}$. 


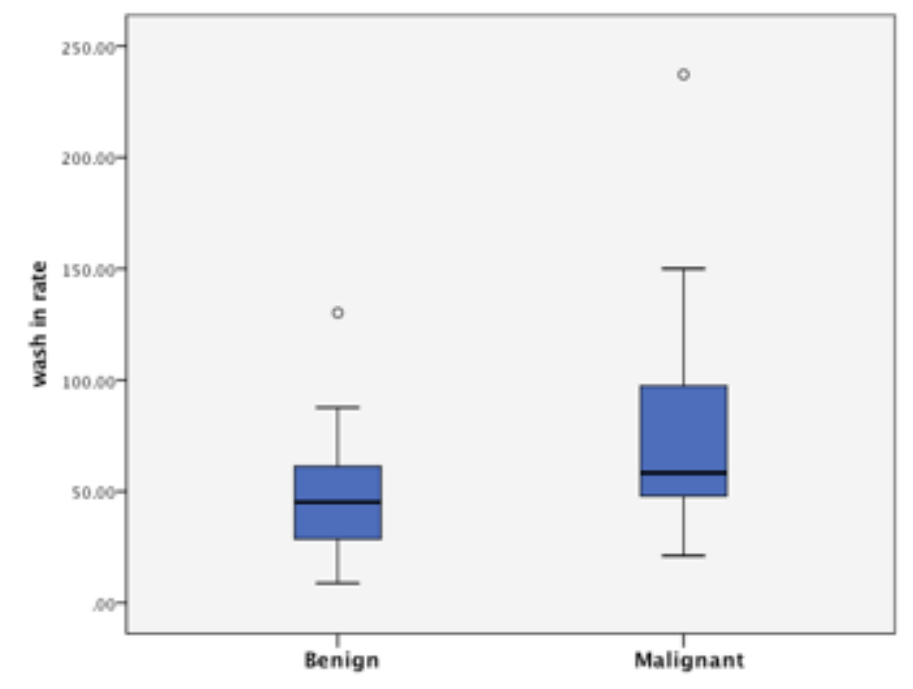

\section{Figure 3}

Box plots show the different kinetic parameters (WIR, WOR, Tpeak, ADC value) in benign and malignant group. The top and bottom of the boxes are the first and third quartiles, respectively. All of the parameters displayed the significant difference $(P<0.05)$.

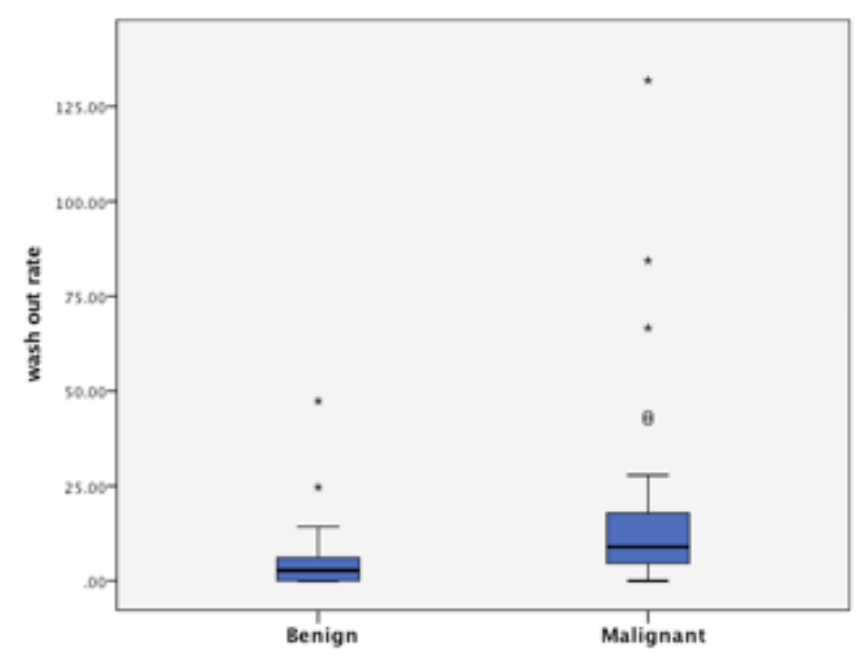

\section{Figure 4}

Box plots show the different kinetic parameters (WIR, WOR, Tpeak, ADC value) in benign and malignant group. The top and bottom of the boxes are the first and third quartiles, respectively. All of the parameters displayed the significant difference $(P<0.05)$. 


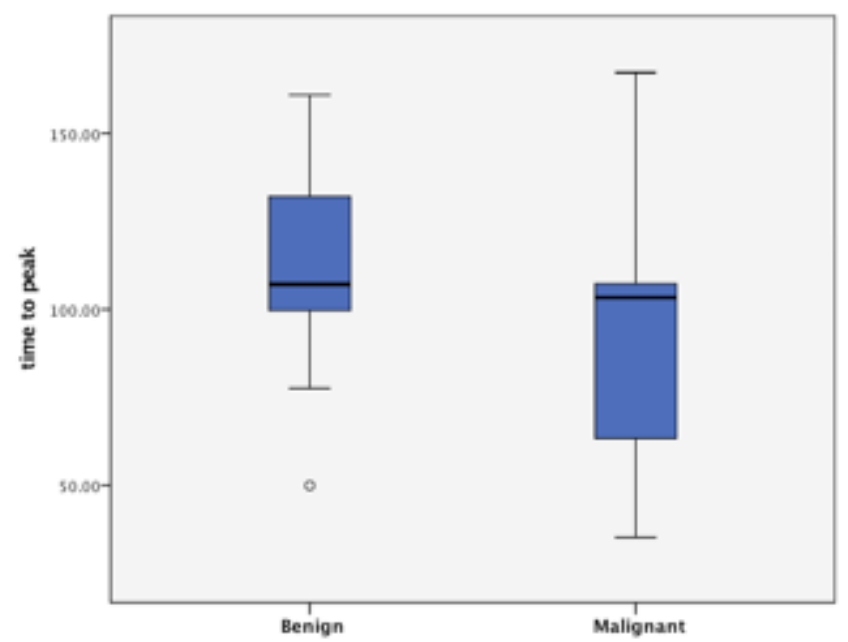

\section{Figure 5}

Box plots show the different kinetic parameters (WIR, WOR, Tpeak, ADC value) in benign and malignant group. The top and bottom of the boxes are the first and third quartiles, respectively. All of the parameters displayed the significant difference $(P<0.05)$.

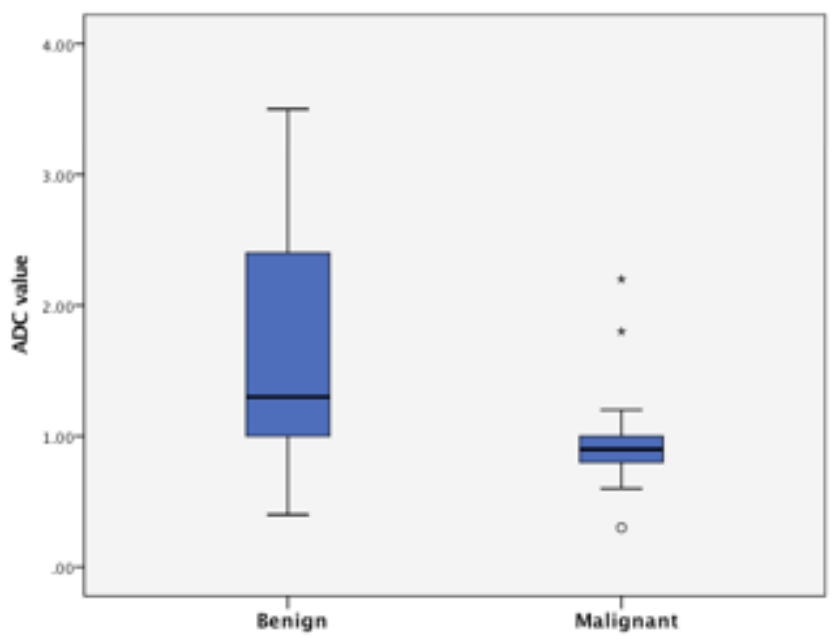

Figure 6

Box plots show the different kinetic parameters (WIR, WOR, Tpeak, ADC value) in benign and malignant group. The top and bottom of the boxes are the first and third quartiles, respectively. All of the parameters displayed the significant difference $(P<0.05)$. 


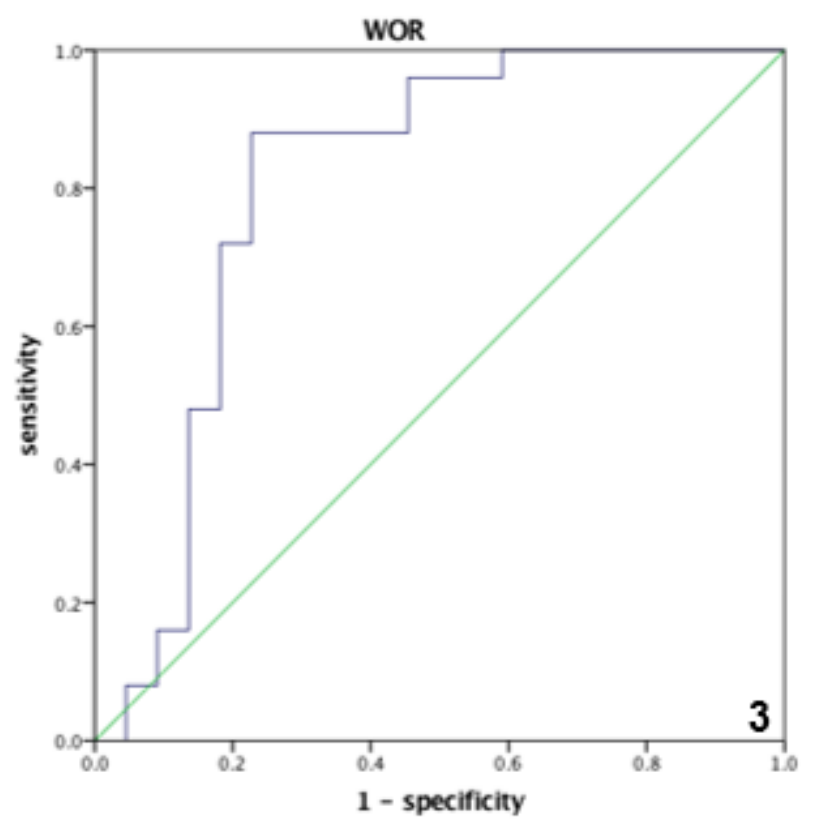

Figure 7

The ROC showed the diagnosis of benign and malignant lesions based on WOR and ADC value demonstrated an AUC of 0.80 , and 0.81 , respectively.

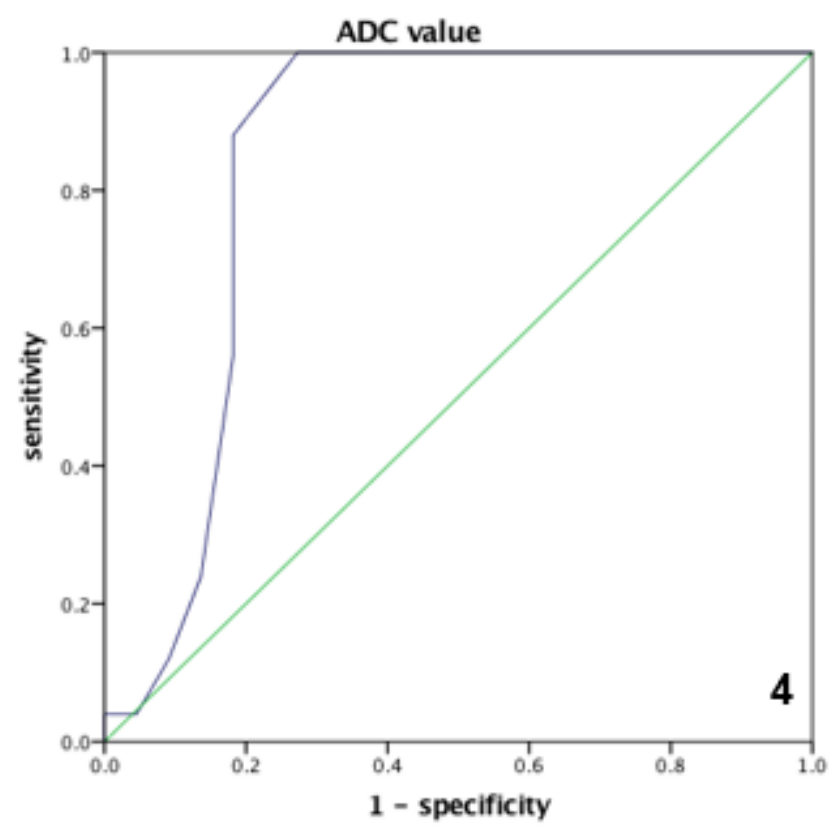

Figure 8

The ROC showed the diagnosis of benign and malignant lesions based on WOR and ADC value demonstrated an AUC of 0.80 , and 0.81 , respectively. 


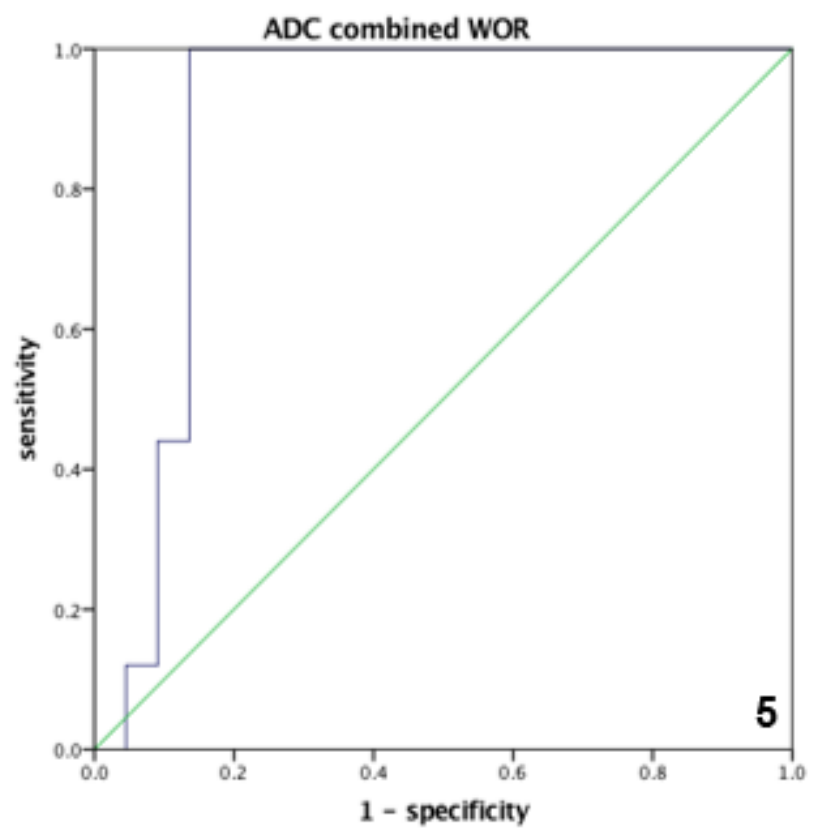

Figure 9

Combination of ADC value and WOR increased sensitivity to $83.30 \%$ and specificity to $87.20 \%$, and the AUC increased to 0.85 . 